\title{
TOPOLOGICAL ENTROPY OF CONTINUOUS SELF-MAPS ON CLOSED SURFACES
}

\author{
JUAN LUIS GARCÍA GUIRAO ${ }^{1}$ AND JAUME LLIBRE ${ }^{2}$ AND WEI GAO ${ }^{1,3}$
}

\begin{abstract}
The objective of the present work is to present sufficient conditions for having positive topological entropy for continuous self-maps defined on a closed surface by using the action of this map on the homological groups of the closed surface.
\end{abstract}

\section{INTRODUCTION}

Along this work by a closed surface we denote a connected compact surface with or without boundary, orientable or not. More precisely, an orientable connected compact surface without boundary of genus $g \geq 0, \mathbb{M}_{g}$, is homeomorphic to the sphere if $g=0$, to the torus if $g=1$, or to the connected sum of $g$ copies of the torus if $g \geq 2$. An orientable connected compact surface with boundary of genus $g \geq 0, \mathbb{M}_{g, b}$, is homeomorphic to $\mathbb{M}_{g}$ minus a finite number $b>0$ of open discs having pairwise disjoint closure. In what follows $\mathbb{M}_{g, 0}=\mathbb{M}_{g}$.

A non-orientable connected compact surface without boundary of genus $g \geq$ $1, \mathbb{N}_{g}$, is homeomorphic to the real projective plane if $g=1$, or to the connected sum of $g$ copies of the real projective plane if $g>1$. A non-orientable connected compact surface with boundary of genus $g \geq 1, \mathbb{N}_{g, b}$, is homeomorphic to $\mathbb{N}_{g}$ minus a finite number $b>0$ of open discs having pairwise disjoint closure. In what follows $\mathbb{N}_{g, 0}=\mathbb{N}_{g}$.

Let $f: \mathbb{X} \rightarrow \mathbb{X}$ be a continuous map on a closed surface $\mathbb{X}$. A point $x \in \mathbb{X}$ is periodic of period $n$ if $f^{n}(x)=x$ and $f^{k}(x) \neq x$ for $k=1, \ldots, n-1$.

The topological entropy of a continuous map $f: \mathbb{X} \rightarrow \mathbb{X}$ denoted by $h(f)$ is a non-negative real number (possibly infinite) which measures how much $f$ mixes up the phase space of $\mathbb{X}$. When $h(f)$ is positive the dynamics of the system is said to be complicated and the positivity of $h(f)$ is used as a measure of the so called topological chaos.

Here we introduce the topological entropy using the definition of Bowen [4].

Key words and phrases. Closed surface, continuous self-map, Lefschetz fixed point theory, periodic point, set of periods.

2010 Mathematics Subject Classification: 58F20, 37C05, 37C25, 37C30. 
Since it is possible to embedded any surface orientable or not in $\mathbb{R}^{4}$ by the Whitney immersion theorem see [11], we consider the distance between two points of $\mathbb{X}$ as the distance of these two points in $\mathbb{R}^{4}$. Now, we define the distance $d_{n}$ on $G$ by

$$
d_{n}(x, y)=\max _{0 \leq i \leq n} d\left(f^{i}(x), f^{i}(y)\right), \quad \forall x, y \in G .
$$

A finite set $S$ is called $(n, \varepsilon)$-separated with respect to $f$ if for different points $x, y \in S$ we have $d_{n}(x, y)>\varepsilon$. We denote by $S_{n}$ the maximal cardinality of an $(n, \varepsilon)$-separated set. Define

$$
h(f, \varepsilon)=\limsup _{n \rightarrow \infty} \frac{1}{n} \log S_{n} .
$$

Then

$$
h(f)=\lim _{\varepsilon \rightarrow 0} h(f, \varepsilon)
$$

is the topological entropy of $f$.

We have chosen the definition by Bowen because, probably it is the shorter one. The classical definition was due to Adler, Konheim and McAndrew [1]. See for instance the book of Hasselblatt and Katok [7] and [3] for other equivalent definitions and properties of the topological entropy. See $[1,2,12]$ for more details on the topological entropy.

Our main results are the following.

Theorem 1. Let $\mathbb{M}_{g}$ be an orientable connected compact surface without boundary of genus $g$. Then the following statements hold.

(a) If the degree $d \notin\{-1,0,1\}$, then the topological entropy of $f$ is positive.

(b) If the degree $d \in\{-1,0,1\}$ and the number of roots of the characteristic polynomial of $f_{* 1}$ equal to \pm 1 or 0 taking into account their multiplicities is not even, then the topological entropy of $f$ is positive.

Theorem 2. Let $\mathbb{M}_{g, b}, b>0$, be an orientable connected compact surface with boundary of genus $\mathrm{g}$. Then the following statements hold.

(a) If $2 g+b-1$ is even and the number of roots of the characteristic polynomial of $f_{* 1}$ equal to \pm 1 or 0 taking into account their multiplicities is not even, then the topological entropy of $f$ is positive.

(b) If $2 g+b-1$ is odd and the number of roots of the characteristic polynomial of $f_{* 1}$ equal to \pm 1 or 0 taking into account their multiplicities is not odd, then the topological entropy of $f$ is positive.

Theorem 3. Let $\mathbb{N}_{g, b}, b \geq 0$, be a non-orientable connected compact surface with boundary of genus $g$. Then the following statements hold.

(a) If $g+b-1$ is even and the number of roots of the characteristic polynomial of $f_{* 1}$ equal to \pm 1 or 0 taking into account their multiplicities is not even, then the topological entropy of $f$ is positive. 
(b) If $g+b-1$ is odd and the number of roots of the characteristic polynomial of $f_{* 1}$ equal to \pm 1 or 0 taking into account their multiplicities is not odd, then the topological entropy of $f$ is positive.

\section{LEFSCHETZ ZETA FUNCTIONS FOR SURFACES}

Let $f$ be a continuous self-map defined on $\mathbb{M}_{g, b}$ or $\mathbb{N}_{g, b}$, respectively. For a closed surface the homological groups with coefficients in $\mathbb{Q}$ are linear vector spaces over $\mathbb{Q}$. We recall the homological spaces of $\mathbb{M}_{g, b}$ with coefficients in $\mathbb{Q}$, i.e.

$$
H_{k}\left(\mathbb{M}_{g, b}, \mathbb{Q}\right)=\mathbb{Q} \oplus n_{k} \cdot \oplus \mathbb{Q}
$$

where $n_{0}=1, n_{1}=2 g$ if $b=0, n_{1}=2 g+b-1$ if $b>0, n_{2}=1$ if $b=0$, and $n_{2}=0$ if $b>0$; and the induced linear maps $f_{* k}: H_{k}\left(\mathbb{M}_{g, b}, \mathbb{Q}\right) \rightarrow H_{k}\left(\mathbb{M}_{g, b}, \mathbb{Q}\right)$ by $f$ on the homological group $H_{k}\left(\mathbb{M}_{g, b}, \mathbb{Q}\right)$ are $f_{* 0}=(1), f_{* 2}=(d)$ where $d$ is the degree of the map $f$ if $b=0, f_{* 2}=(0)$ if $b>0$, and $f_{* 1}=A$ where $A$ is an $n_{1} \times n_{1}$ integral matrix (see for additional details [10, 13]).

We recall that the homological groups of $\mathbb{N}_{g, b}$ with coefficients in $\mathbb{Q}$, i.e.

$$
H_{k}\left(\mathbb{N}_{g, b}, \mathbb{Q}\right)=\mathbb{Q} \oplus n_{k} \cdot \oplus \mathbb{Q}
$$

where $n_{0}=1, n_{1}=g+b-1$ and $n_{2}=0$; and the induced linear maps are $f_{* 0}=(1)$ and $f_{* 1}=A$ where $A$ is an $n_{1} \times n_{1}$ integral matrix (see again for additional details $[10,13])$.

Let $f: \mathbb{X} \rightarrow \mathbb{X}$ be a continuous map and let $\mathbb{X}$ be either $\mathbb{M}_{g, b}$ or $\mathbb{N}_{g, b}$. Then the Lefschetz number of $f$ is defined by

$$
L(f)=\operatorname{trace}\left(f_{* 0}\right)-\operatorname{trace}\left(f_{* 1}\right)+\operatorname{trace}\left(f_{* 2}\right) .
$$

We shall use the Lefschetz numbers of the iterates of $f$, i.e. $L\left(f^{n}\right)$. In order to study the whole sequence $\left\{L\left(f^{n}\right)\right\}_{n \geq 1}$ it is defined the formal Lefschetz zeta function of $f$ as

$$
Z_{f}(t)=\exp \left(\sum_{n=1}^{\infty} \frac{L\left(f^{n}\right)}{n} t^{n}\right)
$$

The Lefschetz zeta function is in fact a generating function for the sequence of the Lefschetz numbers $L\left(f^{n}\right)$.

From the work of Franks in [6] we have for a continuous self-map of a closed surface that its Lefschetz zeta function is the rational function

$$
Z_{f}(t)=\frac{\operatorname{det}\left(I-t f_{* 1}\right)}{\operatorname{det}\left(I-t f_{* 0}\right) \operatorname{det}\left(I-t f_{* 2}\right)},
$$


where in $I-t f_{* k}$ the $I$ denotes the $n_{k} \times n_{k}$ identity matrix, and $\operatorname{det}\left(I-t f_{* 2}\right)=1$ if $f_{* 2}=(0)$. Then for a continuous map $f: \mathbb{M}_{g, b} \rightarrow \mathbb{M}_{g, b}$ we have

$$
Z_{f}(t)=\left\{\begin{array}{l}
\frac{\operatorname{det}(I-t A)}{(1-t)(1-d t)} \quad \text { if } b=0, \\
\frac{\operatorname{det}(I-t A)}{1-t} \quad \text { if } b>0
\end{array}\right.
$$

and for a continuous map $f: \mathbb{N}_{g, b} \rightarrow \mathbb{N}_{g, b}$ we have

$$
Z_{f}(t)=\frac{\operatorname{det}(I-t A)}{1-t} .
$$

\section{BASIC RESUltS}

In this section we present the main result stated in Theorem 7 for proving Theorems 1, 2 and 3. Since its proof is short and important for this work we provide it here.

For a polynomial $H(t)$ we define $H^{*}(t)$ by

$$
H(t)=(1-t)^{\alpha}(1+t)^{\beta} t^{\gamma} H^{*}(t),
$$

where $\alpha, \beta$ and $\gamma$ are non-negative integers such that $1-t, 1+t$ and $t$ do not divide $H^{*}(t)$.

The spectral radii of the maps $f_{* k}$ are denoted $\operatorname{sp}\left(f_{* k}\right)$, and they are equal to the largest modulus of all the eigenvalues of the linear map $f_{* k}$. The spectral radius of $f_{*}$ is

$$
\operatorname{sp}\left(f_{*}\right)=\max _{k=0, \ldots, m} \operatorname{sp}\left(f_{* k}\right) .
$$

The next result is due to Manning [9].

Theorem 4. Let $f: X \rightarrow X$ be a continuous map on a closed surface $\mathbb{X}$. Then $\log \max \left\{1, \operatorname{sp}\left(f_{* 1}\right)\right\} \leq h(f)$.

Lemma 5. Let $f: \mathbb{X} \rightarrow \mathbb{X}$ be a continuous map and let $\mathbb{X}$ be a closed surface. If the topological entropy of $f$ is zero, then all the eigenvalues of the induced homomorphism $f_{* 1}$ are zero or root of unity.

Proof. Since the topological entropy is zero, by Theorem 4 we have $\operatorname{sp}\left(f_{* 1}\right)=1$. So, all the eigenvalues of $f_{* 1}$ have modulus in the interval $[0,1]$ and at least one of them is 1 . Then the characteristic polynomial of $f_{* 1}$ is of the form $t^{m} p(t)$, where $m$ is a non-negative integer, positive if the zero is an eigenvalue. And $p(t)$ is a polynomial with integer coefficients and whose independent term $a_{0}$ is non-zero. Since the product of all non-zeros eigenvalues of $f_{* 1}$ is the integer $a_{0}$ and, these eigenvalues have modulus in $(0,1]$, we have that any of these eigenvalues can have modulus smaller than one, otherwise we are in contradiction with the fact $a_{0}$ is an integer. In short, all the non-zero eigenvalues have modulus one, and consequently $a_{0}=1$. 
Since if a polynomial has integer coefficients, constant term 1 and all of whose roots have modulus 1 , then all of its roots are roots of unity, see [14], the lemma follows.

The $n$-th cyclotomic polynomial is defined by

$$
c_{n}(t)=\prod_{k}\left(w_{k}-t\right)
$$

being $w_{k}=e^{2 \pi i k / n}$ a primitive $n$-th root of unity and where $k$ runs over all the relative primes $\leq n$. See [8] for the properties of these polynomials.

For a positive integer $n$ the Euler function is $\varphi(n)=n \prod_{p \mid n, p \text { prime }}\left(1-\frac{1}{p}\right)$. It is known that the degree of the polynomial $c_{n}(t)$ is $\varphi(n)$. Note that $\varphi(n)$ is even for $n>2$.

A proof of the next result can be found in [8].

Proposition 6. Let $\xi$ be a primitive $n$-th root of the unity and $P(t)$ a polynomial with rational coefficients. If $P(\xi)=0$ then $c_{n}(t) \mid P(t)$.

The proofs of our results are strongly based in the next theorem.

Theorem 7 (Theorem 3.2 of [5]). Let $\mathbb{X}$ be a closed surface, $f: \mathbb{X} \rightarrow \mathbb{X}$ be a continuous self-map, and let $\mathcal{Z}_{f}(t)=P(t) / Q(t)$ be its Lefschetz zeta function. If $P^{*}(t)$ or $Q^{*}(t)$ has odd degree, then the topological entropy of $f$ is positive.

Proof. From the definitions of a polynomial $H^{*}$ and of the Lefschetz zeta function we have

$$
\mathcal{Z}_{f}(t)=\frac{P(t)}{Q(t)}=(1-t)^{a}(1+t)^{b} t^{c} \frac{P^{*}(t)}{Q^{*}(t)}
$$

where $a, b$ and $c$ are integers.

Assume now that the topological entropy $h(f)=0$. Then by Lemma 5 all the eigenvalues of the induced homomorphisms $f_{* 1}$ 's are zero or roots of unity. Therefore, by (1) all the roots of the polynomials $P^{*}(t)$ and $Q^{*}(t)$ are roots of the unity different from \pm 1 and zero. Hence, by Proposition 6 the polynomials $P^{*}(t)$ and $Q^{*}(t)$ are product of cyclotomic polynomials different from $c_{1}(t)=1-t$ and $c_{2}(t)=1+t$. Consequently $P^{*}(t)$ and $Q^{*}(t)$ have even degree because all the cyclotomic polynomials which appear in them have even degree due to the fact that the Euler function $\varphi(n)$ for $n>2$ only takes even values. But this is a contradiction with the assumption that $P^{*}(t)$ or $Q^{*}(t)$ has odd degree. 


\section{Proof of Theorems 1,2 and 3}

Proof of Theorem 1. Since $\mathbb{M}_{g}$ is an orientable connected compact surface without boundary of genus $g$, then the Lefschetz zeta function of $f$ is equal to

$$
Z_{f}(t)=\frac{\operatorname{det}(I-t A)}{(1-t)(1-d t)}
$$

where $d$ is the degree of $f$ and $2 g$ is the dimension of the characteristic polynomial $\operatorname{det}(I-t A)$ of $f_{* 1}=A$. Note here that if $d \notin\{-1,0,1\}$, then $Q^{*}(t)=1-d t$ and therefore by Theorem 7 statement (a) of Theorem 1 is proved.

Assume now that $d \in\{-1,0,1\}$. Note that in this case $Q(t)=(1-t)(1-d t)$ and $Q^{*}(t)=1$. So, by Theorem 7 the main role will be play by the $2 g$ degree polynomial $P(t)=\operatorname{det}(I-t A)$ where $f_{* 1}=A$. Since $2 g$ is even and the number of roots of the characteristic polynomial of $f_{* 1}$ equal to \pm 1 or 0 taking into account their multiplicities is not even, then $P^{*}(t)$ has odd degree. Therefore, statement (b) of Theorem 1 follows by the application of Theorem 7 .

Proof of Theorem 2. Note now, since $\mathbb{M}_{g, b}$ is an orientable connected compact surface with boundary $(b>0)$ of genus $g$, then the Lefschetz zeta function of $f$ is equal to

$$
Z_{f}(t)=\frac{\operatorname{det}(I-t A)}{1-t}
$$

being $2 g+b-1$ the degree of the characteristic polynomial $\operatorname{det}(I-t A)$ of $f_{* 1}=A$. Now the proof is similar to the statements (b) and (c) of Theorem 1 .

Proof of Theorem 3. For a non-orientable connected compact surface with or without boundary $(b \geq 0)$ of genus $g \geq 1$, the Lefschetz zeta function of $f$ is equal to

$$
Z_{f}(t)=\frac{\operatorname{det}(I-t A)}{1-t}
$$

being $g+b-1$ the degree of the characteristic polynomial $\operatorname{det}(I-t A)$ of $f_{* 1}=A$. Then the proof if this theorems follows in a similar way to the proof of statements (b) and (c) of Theorem 1.

\section{ACKNOWLEDGEMENTS}

The second author is partially supported by the Ministerio de Economía, Industria y Competitividad, Agencia Estatal de Investigación grants MTM2016-77278-P (FEDER) and MDM-2014-0445, the Agència de Gestió d'Ajuts Universitaris i de Recerca grant 2017SGR1617, and the H2020 European Research Council grant MSCA-RISE-2017-777911. 


\section{REFERENCES}

[1] R.L. Adler, A.G. Konheim and M.H. McAndrew, Topological entropy, Trans. Amer. Math. Soc. 114 (1965), 309-319.

[2] L. Alseda, J. Llibre and M. Misiurewicz, Combinatorial dynamics and entropy in dimension one, Second edition, Advanced Series in Nonlinear Dynamics Vol. 5, World Scientific Publishing Co., Inc., River Edge, NJ, 2000.

[3] F. Balibrea, On problems of Topological Dynamics in non-autonomous discrete systems, Applied Mathematics and Nonlinear Sciences 1(2) (2016), 391-404.

[4] R. Bowen, Entropy for group endomorphisms and homogeneous spaces, Trans. Amer. Math. Soc. 153 (1971), 401-414; erratum: Trans. Amer. Math. Soc. 181 (1973), 509510 .

[5] J. Casasayas, J. Llibre And A. Nunes, Algebraic properties of the Lefschetz zeta function, periodic points and topological entropy, Publicacions Mathemàtiques 36 (1992), 467-472.

[6] J. Franks, Homology and Dynamical Systems, CBMS Regional Conf. Series, vol. 49, Amer. Math. Soc., Providence R.I., 1982.

[7] B. Hasselblatt and A. Katok, Handbook of dynamical systems, Vol. 1A. NorthHolland, Amsterdam, 2002.

[8] S. LAnG, Algebra, Addison-Wesley, 1971.

[9] A. Manning, Topological entropy and the first homology group, in Dynamical systems - Warwick 1974, Lecture Notes in Math 468, Springer-Verlag, Berlin, 1975, 185-190.

[10] J.R. Munkres, Elements of Algebraic Topology, Addison-Wesley, 1984.

[11] A. Skopenkov, Embedding and knotting of manifolds in Euclidean spaces, , In: Surveys in Contemporary Mathematics, Ed. N. Young and Y. Choi, London Math. Soc. Lect. Notes., 347 (2): 248Đ342, (2008)

[12] P. Walters, An Introduction to Ergodic Theory. Springer-Verlag, 1992.

[13] J.W. Vicks, Homology theory. An introduction to algebraic topology, Springer-Verlag, New York, 1994. Academic Press, New York, 1973.

[14] L.C. Washington, Introduction to cyclotomic fields, Springer, Berlin, 1982.

${ }^{1}$ Departamento de Matemática Aplicada y Estadística. Universidad Politécnica de Cartagena, Hospital de Marina, 30203-Cartagena, Región de Murcia, SPAIN.

E-mail address: juan.garcia@upct.es

${ }^{2}$ Departament de Matemàtiques. Universitat Autònoma de Barcelona, Bellaterra, 08193-Barcelona, Catalonia, Spain

E-mail address: jllibre@mat.uab.cat

${ }^{3}$ School of Information Science and Technology, Yunnan Normal UniverSity, KunMing 650500, China

E-mail address: gaowei@ynnu.edu.cn 